\title{
II. \\ Ein Fall von Mycosis fungoides mit Erkrankung von Nerven und mit Lokalisation in den inneren Organen.
}

\author{
Von \\ Professor Richard Paltauf und Privatdozenten Dr. G. Scherber, \\ Prosektor \\ Primärarzt \\ der k. k. Krankenanstalt „Rudolfstiftung“ in Wien.
}

(Hierzu Taf. I und 4 Textfiguren.)

Durch eine Anzahl in den letzten Jahren mitgeteilter Fälle von Mycosis fungoides. ist sichergestellt worden, daß dieser Erkrankung, die gewöhnlich in der Haut ihre ersten Erscheinungen zeigt, durch die Ausbreitung des Prozesses auf die inneren Organe der Charakter einer Allgemeinerkrankung des Organismus zukommt. Diese gewonnene Erkenntnis zu erweitern, diene die Veröffentlichung des folgenden Falles, der sich an die von Paltauf-v. Zumbusch ${ }^{1}$ ) mitgeteilten Fälle anschließt und weitere neue anatomische Veränderungen darbietet.

Die Private D. S., 60 Jahre alt, wurde am 28. Dezember 1914 auf die Hautabteilung der k, k. Rudolfstiftung aufgenommen. Die jetzige Erkrankung bestand seit ungefähr zwei Jahren und hatte anfangs nur ekzematoiden Charakter. Vor ungefähr einem halben Jahr stellten sich heftige ziehende und reißende Schmerzen in den tieferen Gewebsteilen in der Mitte der Vorderfläche des rechten Oberschenkels ein und diese Beschwerden, die besonders in einem Abschnitt oberhalb des Knies lokalisiert waren, steigerten sich zeitweilig bis zur Unerträglichkeit. Gleichzeitig fingen die an verschiedenen Hautstellen lokalisierten Entzündungsherde stellenweise an in die Höhe zu wachsen und sich zu großen Geschwülsten zu entwickeln.

Status praesens: Die Patientin ist eine große Frau mit anämischer welker Haut, geringem Fettansatz, atrophischer Muskulatur, doch kräftigem Knochenbau. An der äußern Haut sind folgende Veränderungen zu bemerken: die äußere Hälfte beider Wangen ist bis gegen das $\mathrm{Ohr}$ von zusammenhängenden, ziemlich scharf begrenzten, entzündlich roten, im Niveau der Umgebung liegenden Herden eingenommen, die stellenweise reichlich mit kleienförmigen Schuppen gedeckt sind. Mehrere distinkte heller- bis kronengroße ebensolche Herde finden sich in der rechten Schläfengegend und an der linken Stirnhälfte. Das ganze rechte Ohr ist von einem oberflächlichen roten schuppenden Herd eingenommen, und auch an den seitlichen wie an der vorderen Halspartie finden sich einzelne ekzematoide Herde. Eine größere Anzahl kronen- bis fünfkronenstückgroßer Effloreszenzen nehmen einen Teil der Hautoberfläche des rechten Oberarms ein; diese Herde sind alle

1) Mycosis fungoides der Haut und der inneren Organe. Arch. f. Dermat. u. Syph., 118. Bd., 1914. 
bis auf einen der größeren vom Charakter der früher geschilderten; dieser eine größere Herd ist scharf, dabei leicht unregelmäßig begrenzt, tritt über die Hautoberfläche deutlich plateauartig rötlich-bräunlich gefärbt hervor und beim Betasten fühlt man ein dichtes, in die Kutis eingelagertes Infiltrat. In der Mitte der Innenfläche des linken Unterarms sitzt isoliert inmitten gesunder Haut ein ungefähr $6 \mathrm{~cm}$ im Durchmesser haltender kreisrund begrenzter Tumor, dessen Randpartie allmählich bis zu $1 \frac{1}{2} \mathrm{~cm}$ Höhe ansteigt, um sich dann in einem steil abfallenden Kreis zu senken und in einen flachen, schüsselförmigen Substanzverlust überzugehen. Dieser ganze Tumor ist bräunlichrot gefärbt, die zentrale exulzerierte Partie stellenweise graugelb fibrinös belegt. Die Dorsalflächen beider Hände sind von stellenweise konfluierenden, teils völlig. flachen, teils leicht in toto hervortretenden rotvioletten, schuppenden Infiltraten eingenommen. An der Innenfläche des rechten Knies und von hier an der Innenfläche des Oberschenkels hinaufreichend, ein ungefähr handgroßer Tumor, der in seinen untersten Partien sich bis zu $2 \mathrm{~cm}$ Höhe erhebt, sich nach oben gegen den Oberschenkel in toto etwas verflacht und in ein plateauartiges, ungefähr $1 / 2 \mathrm{~cm}$ über die Umgebung vortretendes Infiltrat übergeht. Die Randpartie dieses ganzen mächtigen Infiltrats ist in ungefähr 2-3 cm Breite vom Epithel gedeckt, derb, rotbräunlich gefärbt, stellenweise stärker pigmentiert.

Die epithelisierten Randpartien begrenzen besonders in der distalen Hälfte in wallartiger Form eine unebene, stellenweise tief sich einsenkende Geschwürsfläche, die hie und da rotbräunlich gefärbt und leicht blutend erscheint, dazwischendureh graugelb mißfarbig belegt ist.

An der Haut des rechten Oberschenkels mehrere heller- bis über kronenstïckgroße, scharfbegrenzte, bläulich-braunrote, plateauartig vortretende Infiltrate. Die Haut beider Unterschenkel ist fast völlig von teils isolierten, teils auf größere Strecken konfluierenden bräunlich-violettroten, mäßig schuppenden Infiltraten eingenommen, die sich auch über die Fußgelenke und die proximalen Teile der Dorsa pedis erstrecken. Beide Palmae und Plantae sind frei; die sichtbaren Schleimhärute leicht anämisch, von krankhaften Erscheinungen frei. Die Drüsen im allgemeinen etwas vergrößert, die rechten Leistendrüsen multipel mäßig geschwollen, auf Druck leicht schmerzhaft.

Die interne Untersuchung ergab bei der Aufnahme diffuse bronchitische Erscheinungen leichten Grades über beiden Lungen; am Herzen ein systolisches Geräusch an der Mitralis; der Puls mäßig beschleunigt, zeitweise leicht unregelmäßig. Der Urinbefund normal, W.-R. negativ. Die Untersuchung des Blutes ergibt keine Abweichung vom normalen Befund.

Zur histologischen Untersuchung gelangten von den Hauteffloreszenzen 1. ein flaches, ungefähr kronengroßes Infiltrat der Haut vom rechten Oberschenkel über dem Knie. Dasselbe zeigte folgende Verhältnisse:

Das Epithel ist in der ganzen Ausdehnung des Sehnittes erhalten, die Lamellen des Stratum corneum stellenweise aufgeblättert, an einer Stelle findet sich ein kleines, intraepitheliales $\mathrm{Ab}$ - 
szeßchen, dessen Inhalt eine strukturlose, rötlich gefärbte Masse bildet, die besonders nach oben zu größere und kleinere, rundliche oder mehr längliche Kerne und Kernreste aufweist. Die Keratohyalinschicht des Epithels fast in der ganzen Länge des Schnittes deutlich ausgeprägt, die Zellen des Stratum Malpighii gro8, Kerne und Kernkörperchen meist gut erhalten, nur an einzelnen wenigen Stellen fehlen die Kerne. Die Interzellularräume etwas verbreitert, die Epithelzapfen im ganzen etwas breiter und länger wie normal, die Epithelgrenze nach unten überall gut erhalten. Zwischen den Epithelzellen finden sich stellenweise mehr oder weniger reichlich kleinere, dunkel gefïrbte, runde Zellen. Das Zelleninfiltrat im Bindegewebe entwickelt sich, wie man an den ganz jungen, isolierten Herden in den Randpartien des Schnittes sieht, um die Gefäße der subpapillären Schicht, in Form größerer und kleinerer Herde. Der Hauptherd selbst erstreckt sich tief ins Gewebe, umschließt teilweise die Schweißdrüsen und reicht stellenweise bis ins Fettgewebe. Das Irifiltrat selbst erscheint ödematös, die einzelnen Zellen verschieden weit auseinandergedrängt; zwischen den eigentlichen Infiltratzellen finden sich verschieden reichlich rote Blutkörperchen, die zum Teil in Form und Farbe erhalten, zum Teil unregelmäßig gestaltet und abgeblaßt erscheinen. Was die entzündlichen Zellformen selbst betrifft, so ergibt die Durchsicht der polyhcromen Methylenblaupräparate wie der mit Pyronin-Methylgrün gefärbten Schnitte, daß die Hauptmenge der Zellen von großen, einkernigen Zellen gebildet wird; die Kerne dieser Zellen differieren in ihrer Größe im allgemeinen, einzelne fallen durch ihre bedeutende Größe auf, und diese Formen sind dann meist etwas blasser gefärbt; überall sieht man Mitosen. Neben diesen Zellformen sieht man verschieden große Plasmazellen und stellenweise reichlicher kleine, dunkle Rundzellen. Die elastischen Fasern sind im Bereiche der Infiltration teils völlig verschwunden, teils nur in Resten erhalten; an einzelnen Stellen der Infiltrate kann man die ehemaligen Gefäße an dem ganz oder nur mehr teilweise erhaltenen Elastikaring erkennen.

2. kam zur Untersuchung ein keilförmiges Stüek aus der epithelisierten Randpartie des Tumors vom rechten Vorderarm.

Die Schnitte zeigen hiex eine größere Mächtigkeit des Infiltrats, dessen Zellelemente ungefähr die gleichen sind wie bei dem flachen Herd, nur findet man hier noch mehr Mitosen und stellenweise reichliche Mastzellen. Hier und da Anhäzufungen grüngelblichen Blutpigments.

Hervorzuheben ist noch, daß die jüngsten Herde keine andern Zellformen aufweisen wie die älteren, und daß kein auffälliges Vorhandensein von eosinophilen Zellen in den Herden überhaupt festgestellt werden kann.

Nach Angabe der Patientin bestanden zu Beginn des Leidens die Hauptbeschwerden in dem an die Effloreszenzen gebundenen Juckreiz, der besonders anfallsweise auftrat und sich manchmal zu unerträglicher Heftigkeit steigerte. In den letzten Monaten ließ der Juckreiz im allgemeinen spontan etwas nach, doch wurde Patientin seit ungefähr einem halben Jahr durch neuralgisehe Schmerzen gequält, die in der Tiefe des großen zerfallenden Tumors lokalisiert. waren und sich besonders in der von gesunder Haut gedeckten mittleren Partie des rechten Oberschenkels in der Tiefe fühlbar machten. Dazu traten in den letzten Monaten heftige, besonders anfallsweise auftretende, migräneartige Schmerzen in der rechten Gesichtshälfte, von der Schläfengegend gegen das Auge und gegen die Mitte der rechten Wange ausstrahlend, welche Schmerzen nur durch Morphium für einige Zeit beruhigt werden konnten.

Patientin wurde gleich nach der Aufnahme in Behandlung genommen; sie erhielt Solut. arsenicalis Fowleri in steigender Dosierung und wurden die erkrankten Stellen von Herrn Dr. v. Schmarda mit Röntgenstrahlen bestrahlt, und zwar die 
exulzerierte Partie und die Infiltrate am rechten Oberschenkel am 7. und 28. Januar 1915, der Tumor am linken Arm am 9., 20. und 30. Januar-1915; die linke Gesichtshälfte und der linke Arm wurden am 12. Januar und am 6. Februar, am 23. Januar und am 19. Februar die rechte Gesichtshälfte bestrahlt. Die Einzeldosis betrug je $1 / 2$ bis $3 / 4$ ND. unter $3 \mathrm{~mm}$ Aluminiumfilter. Unter dieser Therapie heilten alle Hauterscheinungen rasch $a b$; die flachen, ekzematoiden Herde reagierten zuerst, aber auch die exulzerierten Tumoren reinigten sich, flachten ab und vernarbten, und Mitte Februar erschienen die früher erkrankten Hautstellen in teils pigmentierte, teils narbig-atrophische Partien umgewandelt. Die Schmerzen in den tieferen Partien des rechten Oberschenkels, die anfangs so heftig waren, ließen unter den Bestrahlungen etwas nach, dagegen blieben die Schmerzen in der rechten Schläfengegend fortbestehen und steigerten sich, gegen das Auge und die Wange ausstrahlend, manchmal in exzessiver Weise. Am 16. Februar zeigte Patientin, nachdem am Vortage heftige Schmerzen in der rechten Gesichtshälfte bestanden hatten, die mit starkem Druckgefühl im Kopf und Brechneigung einhergingen, eine völlige Lähmung des oberen Augenlides und des Sphincter iridis des rechten Auges; es handelte sich um eine peripherische Okulomotoriuslähmung, und wurde als Ursache eine Entzündung des Nerven in seinem Verlauf angenommen. Patientin litt durch die Lähmung besonders schwer psychisch. Gegen Ende Februar nahmen die bronchitischen Erscheinungen über den Lungen zu, Patientin wurde beständig von Hustenreiz gequält. In der Nacht vom 5. zum 6. März kam es bei der Patientin plötzlich zu einer schweren Hämoptoe, die eine völlige Erschöpfung der Kräfte zur Folge hatte; ein gleicher Anfall wiederholte sich in der Nacht vom 7. zum 8. März und am 10. März trat unter allgemeiner Entkräftung Exitus letalis ein.

Die am 11. März vorgenommene Obduktion ergab folgenden Befund:

Körper mittelgroß, kräftig gebaut, kräftige Muskulatur, ziemlich erhaltener Pannikulus, die allgemeine Deçke blaß, an Staimm und oberen Extremitäten von etwas bräunlichem Kolorit durch zahlreiche bräunlich pigmentierte, mohnsamenbis hirsekorngroße Flecke.

Am linken Vorderarm eine ovale, ca. $2 \mathrm{~cm}$ lange bis $2 \mathrm{~cm}$ breite, weißrötliche, im Zentrum stärker gerötete, daselbst narbige Stelle, während in der nächsten Umgebung die Haut verdünnt und zart ist. Die ganze Stelle ist von einem bräunlichen Pigmenthof umgeben. In der linken Inguinalgegend, auf den Mons veneris übergreifend, 3 etwa $4 \mathrm{~cm}$ im Umfang betragende rundliche, konfluierende Stellen, an denen die Haut pigmentiert, zentral atrophisch, mit schuppender Epidermis bedeckt ist. An der Innenseite des linken Kniegelenks ist die Haut etwas geschwollen, an einer regelmäBig ovalen, $2 \mathrm{~cm}$ langen und $3 \mathrm{~cm}$ breiten Stelle der Epidermis entblößt, braunrot, leicht vertrocknet, von einem verheilenden Epidermissaum umgeben. Darüber an der Innen- und Außenfläche je ein bohnengroßer, brauner Pigmentfleck. An der Außenseite des linken Unterschenkels mehrere bräunliche, ovale Herde, von denen der größere zentral schuppende Epidermis zeigt. Am Fußrücken außen links eine ca. $5 \mathrm{~cm}$ im Durchmesser haltende Stelle, an der die Haut etwas verdickt, mit größeren Schuppen bedeckt ist. An der Außenseite des rechten Unterschenkels zwei wenig pigmentierte, rundliche, etwas schuppende Stellen, an denen die Haut zart ist, ebenso an der Außenseite des rechten Fußes. Außen hinten am rechten Kniegelenk neben einer Pigmentierung ein $1 \frac{1}{2} \mathrm{~cm}$ langer und $21 / 2 \mathrm{~cm}$ breiter oberflächlicher Substanzverlust, dessen Rand eine 
blutige Borke trägt, im Zentrum dunkelrot gefärbt; etwas vertrocknet und von einem Pigmentfleck umgeben ist. Innen oben am rechten Kniegelenk eine etwa $21 / 2 \mathrm{~cm}$ lange livide längliche Narbe.

Kopthaar grau. Schädeldach 17,2 cm lang, $1 \overline{0}, 8 \mathrm{~cm}$ größte Breite. Dura mater blaß, innere Meningen zart, am medialen Rande etwas getrübt.

Der rechte N. ocumolotorius gerötet und verdickt. Gehirn mäBig blutrejch, Sulc. etwas erweitert, in den Ventrikeln klares Serum, basale Ganglien blaßgraurot, keine Herderkrankung, die Schwellung und Rötung des Nerv. oculomot. d. setzt sich auf den in der Orbita gelegenen Teil und auf den zum M. rectus inf. ziehenden Ast fort, der deutlich gerötet ist.

Linke Lunge frei; am vorderen Rande nahe einer etwas eingezogenen Stelle bemerkt man eine kleinbohnengroße, blasse, resistente, von einem geröteten Hofe umgebene Stelle, der am Durchschnitt ein ziemlich scharf umschriebener weißrötlicher Tumor entspricht; etwas außen davon findet sich eine kronenstückgroße, nicht scharf begrenzte, gerötete Stelle, die auch resistenter, anf dem Durchschnitt ein dunkelrotes, nicht scharf begrenztes, etwas körniges Infiltrat zeigt; dasselbe hängt mit einem Ast der Pulmonalarterie zusammen; das Lungengewebe der nächsten Umgebung etwas komprimiert. Weiter nach aufwärts tastet man an einer etwas eingezogenen Stelle einen kleinbohnengroßen, im Durchschnitt scharf umschriebenen, fast käsig weißen Tumor, der von schwärzlich pigmentiertem Lungengewebe umgeben ist. Unterhalb der Spitze findet sich an der Iateralen Eläche eine hellerstuickgroße erhabene Stelle, der auf dem Einschnitt ein scharf umschriebener, zentral rötlicher, peripherischer blasser Tumor entspricht. Sonst das Lungengewebe mäßig lufthaltig, in den hinteren Partien blutreicher; in den Bronchien gelbliches, dickschleimiges, fast gallertiges Sekret.

Die rechte Lunge ist im Bereich des Oberlappens frei; der Unterlappen ist groß, konsistent, an seiner Basis mit dem Zwerchfell ziemlich innig verklebt, aber lösbar. Daselbst findet sich an der Pleura diaphragmatica und im Winkel zur Pleura costalis ein $4 \frac{1}{2} \mathrm{~cm}$ langes, $3 \frac{1}{2} \mathrm{~cm}$ breites, fleischrotes, stark erhabenes Infiltrat, das mit dem Unterlappen innig verklebt war; neben diesem findet kostalwärts ein größeres, $2 \mathrm{~cm}$ langes, $11 / 2 \mathrm{~cm}$ breites beetartig erhabenes und ein kreuzerstückgroßes, auch gerötetes oberflächliches Infiltrat. Im Winkel zwischen Mediastinum und Zwerchfell findet sich eine wie aus Konfluenz von mehreren Knoten entstandene fleischrote, etwas knollige, in der Mitte etwa $1 \frac{1}{2} \mathrm{~cm}$ hohe Gewebsmasse. Außer diesen Geschwülsten findet sich an der 9. Rippe ein etwa $4 \mathrm{~cm}$ langes, $2 \mathrm{~cm}$ breites plattes, zentral lebhaft rotes Infiltrat, das von einem blaßroten Saume umgeben ist; an der 11. und 12. Rippe finden sich entsprechend der Axillarlinie kleine, flache, weißrötliche, leicht erhabene Infiltrate (Taf. I). Im Oberlappen findet sich vorn eine $21 / 2$ und $2 \mathrm{~cm}$ im Durchmesser betragende gerötete, von einer zarten Bindegewebsmembran bedeckte resistente Stelle, der entsprechend das Lungengewebe starr, luftleer, karnifiziert erscheint, so daß ein etwa $2 \mathrm{~cm}$ im Durchmesser haltender Anteil sich von dem umgebenden lufthaltigen, 
blassen Gewebe abhebt; in der Tiefe desselben Schnittes findet sich ein kleinbohnengroßer, weißgelber, wie käsiger Herd, der von einer zarten Bindegewebshiulle begrenzt ist; am vorderen Rande unten findet sich an einer leicht eingezogenen, bindegewebig verdickten Stelle entsprechend ein ganz ähnlicher karnifizierter Herd wie der frühere. Der Unterlappen ist, wie bereits erwähnt, groß, starr, konsistent, an der Basis und außen bedeckt von einer hier nur Millimeter, am äußeren Rande und an der Basis aber bis $1 / 2$ cm dicken, lebhaft, stellenweise auch dunkelroten und von Blutungen durchsetzten Gewebswucherung, deren Oberfläche matt, von zarten Fibrinhäutchen bedeckt ist. An der Basis findet sich ein jenem Tumor am Mediastinum-Diaphragma-Winkel entsprechender tiefer Eindruck. Das Gewebe der unteren Hälfte des Unterlappens luftleer, grau und graurötlich infiltriert, undeutlich hepatisiert, an der Basis, den dortigen Infiltraten entsprechend, ohne jede Körnelung, die Schnittfäche glatt, das Gewebe fast homogen, dunkel gerötet, von blasseren, nicht scharf begrenzten hanfkorngroßen Herden durchsetzt. Die Spitze des Unterlappens lufthaltig, schlaff, etwas ödematös, stark pigmentiert, auch die Anteile gegen den Hilus zu etwas lufthaltig, blutreich; nahe der Spitze an einer geröteten Stelle ein kleinhaselnußgroßer, weißrötlicher, ziemlich scharf umschriebener Knoten. In den Bronchien hellgelbes, teils auch eiterähnliches und fibrinös eitriges, zum Teil Ausgïsse bildendes Exudat; die Schleimhaut stellenweise dunkel gerötet:

Die Lymphdrüsen im Hilus hart, stark anthrakotisch, ganz homogen, stellenweise tief schwarz gefärbt.

Im Herzbeutel klares Serum; das Herz entsprechend groß, das subepikardiale Fettgewebe ziemlich reichlich. Über der Basis der Pulmonalarterie das Epikard etwas gerötet, ebenso an der vorderen Wand des rechten Ventrikels, daselbst anch graulich infiltriert. Ferner findet sich am linken Ventrikel entsprechend dem Beginn des Sulcus longitud. das Epikard etwas gerötet, dabei erscheint die Wand daselbst resistenter, links davon eine längliche bohnengroße grauliche Verdickung; beim Durchschnitt zeigt sich die Wand des linken Ventrikels an dieser Stelle und ïbergreifend auf das Septum grau und graweißlich infiltriert, so daß ein nicht scharf begrenzter Knoten von $1-1^{1 / 2} \mathrm{~cm}$ Durchmesser erscheint. Die Klappen zart, das Aortensegel mit einigen weißgelben. Flecken versehen, etwas verdickt, aber schlußfähig.

Die Milz vergröBert, $141 / 2 \mathrm{~cm}$ lang, bei $71 / 2 \mathrm{~cm}$ größter Breite, bis $41 / 2 \mathrm{~cm}$ dick; ihre Kapsel gespannt, das Gewebe dunkelbraunrot, härter, die Follikel undeutlich. Am oberen Pol finden sich in einer dunkelgeröteten Randpartie lichtere, kleinerbsengroße Stellen.

Die Leber kleiner, mit einem deutlichen Schniurlappen versehen; der verhältnismäßig große linke Lappen erscheint durch zahlreiche weißgelbliche, hirsebis hanfkorngroße, durch die Kapsel durchscheinende Herde marmoriert gefärbt. Die Herde sind ganz unregelmäßig, manchmal ganz verzweigt ästchenartig formiert; auch an der Oberfläche des rechten Lappens stellenweise eine solche 
Zeichnung. Am Durchschnitt erscheinen namentlich in der Gegend des Hilus hellbraune und dunklere Partien, wobei die dunkleren leicht eingesunken, atrophisch, die helleren leicht vortretend, wie etwas fetthaltiges Gewebe erscheinen; die Gallenblase groß, in derselben reichliche grüngefärbte Galle.

Beide Nieren mittlerer GröBe, schlaffer, mit zarter Kapsel, Oberfläche glatt, die Rinde blaßgelblich-rot, die Pyramiden dunkler. Nebennieren glatt, ihr Durchschnitt gewöhnlich.

Der Magen ist mehr vertikal gelagert, zusammengezogen; er enthält nur wenig gallige, schleimige Flüssigkeit. Längs der großen Kurvatur erscheinen einige Falten resistenter, dabei grauweißlich, auch stellenweise lebhaft gerötet, infiltriert und starr, so daß sich dieselben fast tu morartig von der Umgebung abheben, auch an der vorderen Wand neben dem Eröffnungsschnitte einige Falten so verändert; sonst ist die Magenschleimhaut dünner, stark gefaltet, blaß; keine Narben aufindbar.

Der Uterus etwas vergrößert, seine Adnexe frei, atrophisch. Die Schleimhaut des Uterus gerötet, von Blutungen durchsetzt, etwas dicker, die Wand $11 / 2 \mathrm{~cm}$ dick. Das Orificium ext. uteri weit, die Zervix kurz, das Orificium int. enge.

Die Harnblase zusammengezogen, zeigt ein kleinfingerweites Divertikel oberhalb der Mündung des linken Ureters; die Schleimhaut blaß, an der hinteren Wand eine scharf umschriebene, braunrote, kreuzergroße, etwas verdickte, im Zentrum grauliche und etwas elevierte Stelle.

Die Schleimhaut des Pharynx und des Larynx blaß, ebenso die der Trachea, die der großen Bronchien leicht gerötet, mit gelblichem schleimigen Sekret bedeckt. Schilddrüse klein, braunrot.

Im Dünndarm spärliche chymöse, im Dickdarm reichliche fäkulente Massen und geformte Skybala. Schleimhaut blaß, im Dünndarm und stellenweise im Dickdarm stark gefaltet; keine Pigmentierungen, keine Narben.

Das Mark des rechten Oberschenkels rot, im distalen Teile gallertig, im proximalen etwas resistenter, hier noch etwas Fettmark.

Das rechte Ganglion Gasseri und auch die eintretende Wurzel, soweit dieselbe im Duralranm gelegen, gerötet. Am rechten Nervus cruralis keine besonderen Veränderungen, außer fleckiger Rötung.

Epikrise: An dem klinischen Bilde des geschilderten Falles ist vor allem der verhältnismäßig kurze Verlauf auffällig; den größten Teil der Krankheitsdauer blieb es nur bei der Entwicklung ekzematoider Erscheinungen und schließlich kam es an einzelnen Stellen auf Basis dieser zur Tumorbildung. Besonders hervorzuheben sind bei unserm Falle die heftigen neuritischen Schmerzen im rechten Oberschenkel, die dem Verlauf des Nervus femoralis entsprachen, wie die von der Schläfe gegen die Stirn und die Wange der rechten Gesichtshälftę ausstrahlenden Schmerzen, welch letztere von besonderer Heftigkeit und migräneartigem Charakter waren und auf ein Befallensein des Nervus trigeminus durch den Krankheitsprozeß 
sehließen ließen; endlich sei der Eintritt der kompletten peripherischen Okulo motoriuslähmung nochmals besonders vermerkt, als deren Ursache sich schon anatomisch eine ausgesprochene Neuritis vorfand, die die nachfolgende mikroskopische Untersuchung als mykosider Natur erwies.

Ferner ist noch die den Krankheitsablauf so beschleunigende, zweimal aufgetretene Hämoptoe zu erwähnen, für die sich eigentlich kein anderes anatomisches Substrat als eine intensive parenchymatöse Blutung (linker Unterlappen) feststellen ließ.

Anatomisch ist die Tumorbildung in der Lunge hervorzuheben, welche in ihrem Charakter völlig den Bildern, die der eine von uns und v. Zumbusch gegeben, entspricht; ferner die Tumoren in der rechten Pleura, die in Form und Farbe so völlig den Hauttumoren entsprechen, daß für sie das in der Bazinschen Beschreibung gebrauchte Vergleichsbild „tomatenähnlich" ganz gut gebraucht werden könnte und in der farbigen Reproduktion dem Betrachter unwillkürlich einfällt. Übereinstimmend mit einem Falle von Zumbuschs ist die Tumorbildung im Magen, dagegen sind Leber, Milz und Lymphdrüsen in unserem. Falle frei. Bemerkenswert sind auch die Knotenbildungen am Herzen; (iiber die an Uterus und Harnblase erhobenen Befunde berichtet der nachträgliche histologische Befund).

Vor Besprechung der histologischen Befunde sei hier besonders mit Rücksicht auf die bestandenen Neurititiden des geschilderten Falles auf einen zweiten Patienten hingewiesen und dessen Krankheitsbild kurz geschildert. Es handelt sich um einen ungefähr 60 Jahre alten Mann, bei dem vor ungefähr einem Jahr an der Außenseite beider Unterschenkel völlig symmetrisch, ungefähr in der Höhe der unteren Grenze des mittleren Drittels, je ein ungefähr guldengroßer, ziemlich scharf umschriebener, entzündlichroter Herd auftrat, dessen Oberfläche stellenweise leicht schuppte; es handelte sich um ein beim Betasten deutlich fühlbares derbes Infiltrat, dessen Bestand von einem ungewöhnlichen Juckreiz begleitet war. Die klinische Diagnose auf Mycosis fungoides wurde durch den histologischen Befund gesichert (Hofrat Paltauf). Die beiden Herde gingen auf eine Röntgenbestrahlungstherapie fast völlig zurück, als vor ungefähr einem halben Jahr, gleichzeitig mit einem Nachschub einiger weniger typischer Effloreszenzen an beiden Armen, sich beiderseits am Dorsum pedis, an den Verlauf des Nervus dorsalis pedis gebunden, heftige neuritische Schmerzen einstellten, die das Gehen fast völlig verhinderten. Es wurde nach einer Arsenkur, die keinen wesentlichen Erfolg hatte, eine neuerliche Röntgentherapie eingeleitet, und Dr. v. Schmarda bestrahlte die Dorsa pedis an den schmerzhaften Stellen mit Tiefenbestrahlung. Der Erfolg war ein auffälliger; die Nervenschmerzen reagierten auf fünfmalige Tiefenbestrahlung, und ist Patient nun schon seit Wochen schmerzfrei. Nach unseren Erfahrungen über die Röntgentherapie der Mycosis fungoides ist ja die Vermeidung der Rezidive sehr zweifelhaft, der zeitweilige Erfolg war jedoch sehr augenseheinlich und für den Patienten von bedeutendem Nutzen. 


\section{Histologische Untersuchung der Leichenorgane.}

Von den Hautläsionen beansprucht der Befund an dem Substanzverlust an der Innenseite des linken Kniegelenkes ein gewisses Interesse, weil er den Vorgang der Rückbildıng mykosider Infiltrate illustriert.

Es fehlt das Papillarstratum und liegt die Kutis blob, die in den oberen Schichten zwar noch von atrophischen Zellen, meist nur pyknotischen Kernen infiltriert ist, deren Balken aber doch bereits deutlich sind und sozusagen zusammensintern; ein Granulationsgewebe fehlt. Von mykosidem Gewebe sind nur einige Inseln an der Grenze der Kutis und Subkutis teilweise erhalten. Die nächste Umgebung des Geschwürs zeigt am Rande verzweigte interpapillare Retezapfen, die Kapillaren der Papillen und die Gefäße des subpapillaren Stratums. stark erweitert, strotzend mit Blut gefüllt, die Papillen verbreitert, ihr Gewebe sowie das der angrenzenden Schicht besteht aus einem fast leeren, zarten, bindegewebigen Retikulum, in dem sich fast nur pyknotische Kerne, Pigmentzellen und ganz vereinzelt noch Zellen mit deutlichem Protoplasma, aber auch mit dunklen, länglichen oder unregelmäßigen Kernen finden - also der Befund eines schwindenden Infiltrates, wie es der eine von uns vor Jahren bereits an einem schwindenden Knoten erhoben hat ${ }^{1}$ ). Die Epidermis zeigt am Gesehwürsrand starke Verhornung und legt sich mit einem Zapfen Retezellen über das mit Zerfall des Infiltrates kollabierende Kutisgewebe.

Die histologisehe Untersuchung der versehiedenen inneren Lokalisationen, sowohl der tumorartigen Bildungen in der Lunge und an der Pleura als der Infiltrate im Herzmuskel und in der Magenwand, nicht minder der makroskopisch bereits veränderten Nervenstämme wie des N. oculomotorius und N. trigeminus ergab einheitliche Veränderungen, nämlich die Entwicklung des der Mycosis fgd. zukommenden charakteristischen Gewebes in verschiedener Menge und Ausbildung; auch im Nervus cruralis d. fanden sich analoge Veränderungen, wie in den genannten makroskopisch veränderten Nerven.

Vielfach waren in diesem Falle die Gewebe sehr gut erhalten; überall trat die eigenartige Zusammensetzung des mykosiden Gewebes aus verschiedenen größeren und kleineren Zellen deutlich hervor, ja die Spezifizität des Gewebes ist in manchen Herden, wie namentlich der Lunge, und in den Veränderungen der Nerven vielleicht noch mehr in die Augen springend, als es jeweils in den Infiltraten und Tumoren der Haut der Fall zu sein pflegt. Namentlich sind die sogenannten großen Uninukleären, die nicht nur eine große Verschiedenheit in Größe und Kontur ihres Leibes, sondern auch der Kerne zeigen, sehr schön ausgebildet. Im allgemeinen durch deutlichen Protoplasmaleib und 2-3 große helle Kerne ausgezeichnet, zeigen sie hier oft einen großen, vielgestalteten Protoplasmaleib mit scharfer Begrenzung, so daß sie mit ihren hellen großen Kernen sehr an epitheloide Zellen erinnern,

1) Paltauf, R., Über lymphatische Neubildungen der Haut. II. intern. Kongr. f. Dermat. u. Syph, 1892. 
häufig aber mehrfache und lange Fortsätze, so daß sie eher Fibroblasten gleichen und bei exzentrischer Lage des Kernes flügelartiges Aussehen gewinnen. Dabei lassen sich in manchen Objekten die Formen ihrer Degeneration und ihres Absterbens verfolgen: sie verlieren den deutlichen Kontur, das Protoplasma bröekelt sich ab, es erscheint von einzelnen Vakuolen durchsetzt, von schaumiger Struktur oder zerklüftet, die Kerne werden kleiner, pyknotisch, verschwinden ganz, und es bleiben kernlose, manchmal etwas hyaline Schollen noch übrig; eine andere Art der Degeneration ist Quellung unter Verdichtung der Peripherie, so daß kuglige Gebilde mit einem hyalinen Randsaum und hellem Innern resultieren. Die Ähnlichkeit mit Fibroblasten wird dadurch gesteigert, daß die Zellen phagozytieren; weiße, auch rote Blutkörperchen, Kohlen- und Hämosiderinpigment (Lunge), Fettkügelchen (Nerv) finden sich in ihnen, doch keine Zeichen einer Weiterentwicklung. In der Lunge ist ihre Differenzierung von wirklichen Fibroblasten bei den neberiher gehenden Vorgängen von Organisation der Exsudate ebenso schwer wie ihre Unterscheidung von gequollenen desquamierten Alveolarepithelien.

Lunge. In einem etwa taubeneigroßen Knoten der Lunge, der einen mittleren, knorpelfreien Bronchus im Längsschnitt und mehrere seiner Verzweigungen in Querschnitten enthält, finden sich ziemlich frische Veränderungen: die Sehleimhaut und die bindegewebige Wand der Bronchien ist von mykosidem Gewebe infiltriert, in welchem man noch auseinandergedrängte Muskelfasern erkennt, das Epithel fehlt oder ist abgestoßen am Rande der nur zum Teil Zellen enthaltenden, meist zellarmen, aus mit Eosin gefärbten grobfädigen oder schleimigen Massen bestehenden Sekretpfröpfe; die großen Zellen in den verschiedensten Formen der Ausbildung und auch des Absterbens, in den tieferen Schichten enthalten dieselben auch ein braunschwarzes Pigment. Das mykoside Gewebe der Bronchien hängt zusammen mit solchem um die Blutgefäße (Arterien), wobei auch ihre Adventitia infiltriert ist, anderseits greift es rom peribronchialen Zellgewebe auf die Wand der anliegenden Alveolen, die größeren Septa über und dringt auch in die Alveolen ein. Diese zeigen außer gelegentlich blutigem Inhalt ohne sonstige Veränderung zahlreiche gequollene Epithelien oder fädig hyaline Massen mit wenig Zellen, zumeist jedoch diese bereits durchsetzt oder umwachsen von einem dem mykosiden ganz ähnlichen Gewebe: fädig körnige Massen mit eingelagerten großen, an Protoplasma reichen, vielgestaltigen Zellen und mehr weniger zahlreichen kleineren und kleinen Rundzellen mit einfachen runden Kernen; vereinzelte zeigen die Eigensehaften der Plasmazellen, multinukleäre Leukozyten sind spärlich. Während an manchen Stellen, namentlich an den zentralen Anteilen, durch die Verbreiterung des peribronchialen und perivaskulären Bindegewebes durch die Wucherung im interstitiellen Bindegewebe und in den Alveolarräumen, solche nur mehr vereinzelt und als schmale Räume zu erkennen sind, erscheinen solche an der Peripherie noch deutlich neben der verdickten und zellreichen Wand erhalten, mit fädigen Massen und desquamiertem Epithel oder mit mehr körnigen und scholligen und mehr weniger zahlreichen Rundzellen erfiillt. 
Sehr schön und markant findet sich die interstitielle Wucherung myk0siden Gewebes an einem Schnitte aus den diffus affizierten Partien des linken Unterlappens, indem hier das perivaskuläre, um die größeren wie kleineren Gefäße das peribronchiale, interlobuläre Bindegewebe sowie die verdickten Alveolarwandungen vo mykosidem Granulationsgewebe durchsetzt sind, während die Alveolarräume mit körnigen oder fädigen Fibrinmassen und Zellen, häufig auch mit großen desquamierten gequollenen Alveolarepithelien erfüllt sind, an der Alveolarwand die großen, glatten, seltener etwas kubischen Epithelien sich finden. Das Bild erinnert in hohem Maße an das einer interstitiellen Pneumonie, zudem auch die ersten Anfänge einer Organisation der Exsudate nicht fehlen, nur mit dem Unterschiede, daß nicht junges Bindegewebe, sondern mykosides Gewebe proliferiert.

Gewinnt man an solchen Präparaten bereits den Eindruck, daß es durch zụnehmende Gewebswucherung zur vollständigen Aufhebung des Lungenparenchyms, auch zum Verschluß kleinerer Bronchien kommt, so zeigt dies in augenscheinlicher Weise ein im Zentrum grauweißlicher, ziemlich umschriebener Knoten. Derselbe ist unter der Pleura gelegen, von der Größe einer Haselnuß. Dieser zeigt in der Mitte einen mit zellarmem Schleim erfültten Hohlraum, der von mykosidem Gewebe rings umschlossen ist, keine epitheliale Auskleidung besitzt, aber durch vereinzelte Muskelfasern in den Innenschichten sich, abgesehen von seinem Inhalt, als Bronchus erkennen läßt, weiter einen größeren Ast der Pulmonalarterie, dessen Wand fast ganz von mykosidem Gewebe infiltriert ist, während sein Lumen teilweise von einem Plättchen- und Leukozytenthrombus erfüllt ist; der zunächst anliegende Anteil des Knotens ist nekrotisch, an andern Stellen fanden sich im mykosiden Gewebe unregelmäBige Herde aus körnig-fädigem Fibrin mit Kerntrümmern, den Resten von Alveolarexsudaten. Der Knoten erscheint durch eine Zone von in interstitieller und organisierender Entzündung begriffenen Alveolen ziemlich scharf von komprimiertem Lungengewebe abgegrenzt, dessen spaltförmige oder längliche Alveolen bei normaler Wand desquamiertes Epithel enthalten. Die interstitiell pneumonischen Veränderungen reichen bis an die etwas verdickte Pleura, unter der sich mit kubischem Epithel ausgekleidete Alveolargänge finden. Dabei verschwindet das elastische Gewebe der Alveolen sehr bald und bleibt zumeist nur das der Gefäße, auch der Bronchien, erhalten; während in erst beschriebenen rezenten Infiltrate dasselbe noch erhalten ist, erseheint es in dem letzteren nur noch an der Peripherie, entsprechend den erhalten gebliebenen, komprimierten Alveolen nachweisbar.

Während in diesem Knoten, nach der peripherischen Abgrenzung zu schließen, das Wachstam sich dem Abschluß nähert, zeigt der kleinbohnengroße weißgelbe wie käsige Knoten im l. Oberlappen dasselbe eingetreten; mikroskopisch erwics sich derselbe ganz nekrotisch, ohne jegliche Kernfärbung, dicht umschlossen von einem teils spindelzelligen, teils auch fasrigen Bindegewebe mit zahlreichen Epithelkanälchen, in welchem keine Spur von mykosidem Granulationsgewebe zu finden 
ist; nur in der nekrotischen Masse erkennt man neben fädigen Netzen noch die Schollen der großen Uninukleären, - - dagegen zeigen Schnitte vom hinteren unteren Lungenrande den Prozeß trotz teilweiser völliger Infiltration des Lungengewebes an der Pleura noch in weiterer Entwicklung. Der Schnitt hat eine dreieckige Form mit abgerundeter Spitze. Zunächst läßt sich das Lungengewebe gar nicht mehr erkennen, nur an der Basis fanden sich noch einige mit Exsudat erfüllte Alveolen, sonst sieht man nur ein sehr zellreiches, aus verschiedenen Zellen zusammengesetztes, im Vergleich mit den Randpartien wenig vaskularisiertes Gewebe. Die Zellen sind verschieden groß, haben meist etwas hellere Kerne, zeigen häufig Mitosen und sind in ein zartes Netzwerk eingelagert; wo sie recht zahlreich sind, erscheinen sie gleichartiger, größer als Lymphozyten, an andern Stellen finden sich auch die größeren Formen, wie sie oben beschrieben sind; ị der Mitte ist ein größerer Arterienast teilweise im Längsschnitt getroffen, dessen Wand fast ganz in das zellreiche Gewebe aufgegangen, während das Endothel durch ein unter demselben. gelagertes lockeres Gewebe abgehoben ist, das aus einem zarten Retikulum und kleineren Zellen mit runden und ovalen Kernen besteht, unter denen die erwähnten epitheloiden oder runden wie ödematösen nicht fehlen. Bei der Elastikafärbung: erscheint diese stark aufgefasert, die Lamellen auseinandergedrängt und finden sich kleinere, völlig oder fast völlig verschlossene Gefäße, deren Elastika weit auseinandergedrängt, zwischen ihren Lamellen und im Lumen ist dasselbe mykoside Gewebe entwickelt, oder es ist durch Thromben verschlossen ${ }^{1}$ ); einige sind auch samt ihrer nächsten Umgebung nekrotisch. An beiden Längsseiten findet sich nun eine durch zahlreiche, vielfach senkrecht auf die Oberfläche gerichtete, strotzend mit Blut gefüllte, weite Gefäße ausgezeichnete, bei 1/2 $\mathrm{cm}$ dicke Gewebsschicht, welche, wie die Elastika- und auch die van Gieson-Färbung zeigt, der Pleura aufgelagert ist, an der Oberfläche in horizontal verlaufende, verschieden dicke Fibrinfäden, Fibrinnetze und Lamellen endet. Diese Schicht ist verschieden zellreich; die zellärmeren Partien zeigen zwischen den weiten Blutgefäßen ein zartes Netzwerk mit großen, protoplasmareichen, vielgestaltigen Zellen, die teils vereinzelt oder dichter gelagert sind, epitheloiden Zellen gleichen, deren Kerne verschieden, die großen hell, die kleinen dunkel gefärbt sind; andere, namentlich die tieferen Partien, sind sehr zellreich und von dem die Lunge durchsetzenden Gewebe nur an den Resten des Bindegewebes und der elastischen Fasern der Pleura abzusondern. Dieses zellreiche Gewebe entspricht mit seimem Retikulum, den verschiedenen Zellen mit Protoplasmazerfall, den eingelagerten Körnern, auch Chromatinpartikeln ganz dem mykosiden. Die oft sehr weiten Blutgefäße haben nur kapillären Ban; einzelne sind thrombosiert; die Thromben bestehen entweder nur aus roten Blutkörperchen, die innig verklebt, einzeln nieht mehr differenzierbar sind, oder auch aus Fibrin; in ihrer Umgebung finden sich Blutungen im Gewebe, auch Zellnekrosen.

1) Vgl. Fig, 7 u. 8 Taf. XXV im 118. Bande des Arch. f. Dermat. u. Syph. 
Fibrinfärbungen der beschriebenen Schnitte zeigen solches nur in den Alveolen, wobei sich namentlich Reste solcher im sonst zellreichen Gewebe sehr deutlich abheben, teilweise in den fädigen Anteilen der Bronchialpfröpfe, in den Gerinnseln der Blutgefäße und in den obersten Sehichten der Pleuraauflagerung, stellenweise noch in den zellarmen Anteilen dieser. Das zarte Netzwerk im mykosiden Gewebe, so auch der zellreichen Anteile der Auflagerung, gibt die Farbreaktion nicht.

Wie es die histologischen Befunde zeigen, schließen sich die mykosiden Knoten. und Veränderungen im vorliegenden Falle vollkommen denen von einem von uns mit Zumbusch beschriebenen an, nur fehlen hier die ausgedehnten Nekrosen und Zerfallsherde; namentlich sind die an den Blutgefäßen erhobenen Veränderungen so identisch, daß man die Präparate der älteren Fälle und des jetzigen Falles nicht zu unterscheiden vermag.

Knoten der Pleura. Während die Wucherungen an der Lungenpleura den Vorgang der Organisation eines fibrinösen Exsudates nachahmen, stellen die Knoten und Gesehwülste an der Pleura parietalis, sowohl der kostalen als der des Diaphragmas, Wucherungen in derselben vor; es wurden zwei derselben, ein kleinerer, flach gewölbter, und der große, fast zapfenförmige, untersucht. In beiden Fällen finden sich ausgedehnte, sehr zellreiche Infiltrate, welche zunächst in lockeren Anteilen des subpleuralen Bindegewebes entwickelt, stärkere Bindegewebszüge freilassen, so daß selbst der größere Tumor zwischen Herden sehr zellreichen Gewebes noch solche zeigt. Die Oberfläche wird noch von dünnen Lagen der obersten Bindegewebsschicht bedeckt. Die Zellanhäufungen erinnern in ihrer Zusammensetzung am meisten an die der Haut, indem sie aus den verschiedenen größeren und kleineren Rundzellen bestehen; es treten aber die großen, vielgestaltigen Uninukleären bei den meist sehr zellreichen Gewebsformen ebenso zurück, wie es auch in den Hauttumoren wiederholt der Fall ist. Bei den flachkugligen Knoten am Diaphragma greift die dichte Zellwucherung durch die Faszie auch auf die angrenzende Muskulatur über, deren oberflächlichste Schicht stellenweise infiltriert ist. An solchen Stellen fortschreitender Erkrankung sind Lockerung des Zellgewebes, Ödem, auch feinste netzige Gerinnung, Gefäßfülle die ersten Veränderungen, welche mit dem Auftreten großer Uninukleärer und kleiner Rundzellen, auch multinukleärer, verbunden sind.

Herz. Die Infiltrate am Epikard und im Herzmuskel bestehen ebenfalls aus mykosidem Granulationsgewebe, das nicht nur den größeren Bindegewebszügen folgt, sondern auch zwischen die Muskelzellen dringt und dieselben auseinanderdrängt. An den Grenzen sieht man auch hier Auflockerung und Auffaserung des Bindegewebes mit Ödem und feinsten Fibrinnetzen der Zellwucherung vorausgehen; die großen Zellen zeigen die verschiedensten Formen, phagozytieren reichlich multinukleäre Leukozyten, erscheinen hydropisch gebläht oder besitzen ein auch mit Pyronin stark färbbares Protoplasma. Wo der Zellreichtum größer ist, haben wir wieder die verschieden großen, zwischen den Muskelzellen häufig etwas ovalen; länglichen Zellen, auch Plasmazellen, Lymphozyten und vereinzelte Leukozyten. 
Die Muskelfasern werden weit auseinandergedrängt, erscheinen sowohl als längliche, faserartige, als auch als unregelmäßige Gebilde, an denen lange die Struktur, Längs- und Querfaserung erhalten bleibt; sehr deutlich ist das Retikulum entwickelt, das, wie die Färbung nach Mallory zeigt, mit dem normalen zarten Bindegewebe zusammenhängt. Zum totalen Verschwinden der muskulösen Elemente ist es nirgends gekommen; am weitesten in die Tiefe reichen die Veränderungen an stärkeren Bindegewebsziugen.

Wie sich die Veränderungen in der Lunge eng an die von einem von uns und Zumbusch beschriebenen anschließen, so ist es auch mit denen der Magenschleimhaut der Fall, doch sind dieselben im vorliegenden Falle bei weitem nicht so entwickelt und beschränken sich auf eine in den unteren Schichten der Mukosa entwickelte und auf die Submukosa übergreifende Infiltration mit mykosidem Gewebe, ähnlich wie die am Epikard, und doch findet sich auch hier ein durch mykoside Gewebsinfiltration schwer veränderter Arterienast.

Im Fundus uteri fand sich die Schleimhaut an der hinteren Wand intensiv gerötet und von Blutungen durchsetzt; ein Stück der hinteren Wand, wo die Veränderung namentlich ausgesprochen war, wurde zur histologischen Untersuchung konserviert. Dieselbe ergab, daß auch hier eine Lokalisation mykosiden Gewebes vorlag. Die Schleimhaut ist sehr aufgelockert, ödematös, hyperämisch, von frischen Blutungen durchsetzt. Das Epithel der Oberfläche erhaiten, besteht aus verhältnismäßig großen, fast kubischen, teilweise fimmernden Zellen. Die Drüsen sind nicht zahlreich, weit. Die Grenze der Schleimhaut und Muskularis vollständig verwischt, indem, wie in der Schleimhaut, ein zellreiches, aber doch lockeres, von sehr weiten, strotzend mit Blut gefuillten Gefäßen durchzogenes Gewebe entwickelt ist, welches die Muskulatur der inneren Schichten zum Teil nur in Bündel, zumeist in ganz vereinzelte Züge auseinandergedrängt hat. Dieses Gewebe enthält wieder in ausgesprochener Weise die vielfach beschriebenen Mykosidzellen in allen Formen ihrer Ausbildung und Rückbildung mit den kleineren und kleinsten Elementen von der Art der Lymphozyten in einem Retikulum, welches ungleichmäßig und nicht so zart ist, wie an andern Orten. Die mykoside Gewebsinfiltration reicht bis etwa in die Mitte der Muskularis, wo sich die größeren, mit starken endarteriitischen Veränderungen versehenen Gefäße finden.

Die Schleimhaut der Harnblase zeigt an der makroskopisch veränderten Stelle starke Füllung sowohl der obersten subepithelialen Kapillaren als auch der tieferen Gefäße, Auffaserung der obersten Schicht, stellenweise Ödem, Leukozyten und Einlagerung größerer uninukleärer Zellen, die an Epitheloidzellen erinnern, Plasmazellen und in der anliegenden Submukosa Riesenzellen; diese besitzen 4-8 Kerne, liegen in den Spalten des Bindegewebes oder an der Wand von spaltförmigen Hohlräumen, an denen man aber keine zellige Auskleidung nachweisen kann. Das Oberflächenepithel ist lädiert, es sind nur die untersten Schichten erhalten, doch scheint ein Artefakt (Manipulation) bei und nach der Obduktion die Ursache zu sein. Nach der Art der Gewebsveränderung dürfte kein Zweifel bestehen, 
daß es sich auch um eine noch oberflächliche und im Anfang stehende mykoside Gewebsbildung handelt.

Die untersuchten inguinalen Drüsen zeigten Fettgewebe im Hilus, eine follikelreiche Kortikalis auch mächtige Follikularstränge, überall ist aber der Bau erhalten, nur die Zeichen einer erhöhten funktionellen Tätigkeit sind als Ursache ihrer klinisch konstatierten leichten Vergrößerung anzunehmen. Auch eine vergrößerte, stark anthrakotische Bronchialdrüse bot in den micht indurierten Anteilen nur starke Desquamation des Endothels in den erweiterten, noch erhaltenen Lymphwegen.

Die mikroskopische Untersuchung der Leber zeigte zahlreiche atrophische, mit weiten Kapillaren versehene Anteile, zwischen denen andere vergrößerte, auch große zweikernige Zellen, etwas Fettinfiltration aufweisen; an günstigen Stellen erkennt man, daß im selben Azinus die zentralen Anteile atrophisch sind, während die peripherischen hypertrophiert sind; die atrophischen Leberzellen bilden nur schmale, pigmentierte Balken zwischen weiten, strotzend mit Blut gefüllten Kapillaren; es finden sich aber auch noch Herde nekrotischer Leberzellen, wobei aber sonstige Veränderungen fehlen. Das interstitielle Bindegewebe ist ganz zart, nirgends im periportalen Gewebe auch nur eine Spur von Zellvermehrung, ebenso fehlt eine solche in den atrophischen Teilen. Mallory-Färbung zeigt deutlich, wie an diesen Stellen das Stützgewebe entsprechend dem Zellausfall zusammengeriuckt ist. Da sonst Zeichen einer passiven Hyperämie fehlen, die Zentralvenen auch der atrophischen Partien offen sind, so ist eine vaskuläre Ursache für diese zentrale Atrophie ausgeschlossen. Es muß ein toxisches Agens die Ursache sein, womit das Vorkommen insulärer Leberzellnekrosen im Einklang steht; worin dasselbe besteht, ob in den therapeutischen Maßnahmen (Röntgenbestrahlung) oder im Krankheitsprozeß selbst, läßt sich nicht entscheiden. Die Veränderung ist am stärksten im l. Lappen und überhaupt in den subkapsulären Schichten; in der Tiefe des rechten Lappens sind ausgedehnte Partien braungelb, ohne die Sprenkelung, wie es nachträgliche Inspektion der im großen konservierten Anteile gezeigt hat.

Auch die Milz zeigt keine mykosiden Veränderungen; ihre Pulpa ist außerordentlich blutreich, sehr zahlreich sind in derselben Zellen, welche mit roten Blutkörperchen erfüllt sind; die Follikel erscheinen unregelmäßig, häufig vergrößert, bestehen zum kleineren Teil aus kleinen Lymphozyten, zahlreicher sind größere Zellen mit deutlichem Protoplasma; die Arterienästchen der Follikel zeigen häufig hyaline Degeneration der Media. Auch die makroskopisch lichteren Stellen am. oberen Pol zeigen nur größere Follikel. Die Färbung mit Pyronin-Methylgrün zeigt zahlreiche große und kleinere Plasmazellen in den Follikeln, aber auch zerstreut und in Gruppen in der Pulpa.

Die Veränderungen an den Nerven; N. oculomotorius. Das intrakranielle Stück des Nerven wurde in Osmiumsäure fixiert, in Alkohol nachgehärtet, die Schnitte mit Saffranin gefärbt. Die in ihrer Markscheide schwarz gefärbten Nervenfasern erscheinen entsprechend der makroskopisch sichtbaren Verdickung 
und Rötung durch um strotzend gefüllte Blutgefäße angeordnete Zellanhäufungen auseinandergedrängt (Textfig. 3). Diese bestehen aus größeren, vielgestaltigen Zellen mit reichlichem Protoplasma und meist hellen Kemen und kleineren uninukleären Rundzellen bis zur Größe von Lymphozyten, neben welchen mit dunklem, rundem Kern auch solche mit unregelmäßigen Kernen nicht fehlten. Wie oben angeführt, sind die großen, vielgestaltigen Uninukleären in allen ihren verschiedenen



Fig. 3. Längssehnitt durch den erkrankten Teil des N. oculomotorius, Fixation in Osmiumsäure, Färbung mit Saffranin. Die Nervenfasern durch das um Gefäße entwickelte mykoside Gewebe auseinandergedrängt; in denselben zahlreiche große Zellen neben kleineren Rundzellen. Fetttröpfehen vom Zerfall der Markscheiden. Vergx. 1:80.

Formen hier sehr ausgesprochen, und wäre die obige Beschreibung nur za wiederholen; sie finden sich in den ersten Zellvermehrungen; in dem noch unveränderten Nerven mit den dicht gelagerten Nervenfasern treten diese Zellen bereits auf. Die Nervenfasern bleiben zunächst unverändert, dann zeigt sich Schwellung der Markscheide, aber erst in den größeren Infiltrationsherden, wie in dem der unteren Hälfte der Abbildung; tritt tropfiger Zerfall auf; da beteiligen sich außer 
kleineren Zellen auch die großen Uninukleären an der Phagozytose der Fettröpfehen. Immer ist die Entwicklung des mykosiden Gewebes von starker Gefäßfüllung begleitet, wie es auch den Gefäßen folgt und häufig von einem Ödem mit Lockerung und Auffaserung des endoneuralen Bindegewebes eingeleitet wird. Die Achsenzylinder bleiben auch bei Zerfall der Markscheide erhalten.

Ganz analog sind die Veränderungen im $\mathbb{N}$. trigeminus; auch hier finden sich zuerst lockere Zellansammlungen von großen, vielgestaltigen Uninukleären und Lymphozyten in einem zarten, grobmaschigen Retikulum, welche dichter werden und dann aus kleineren Uninukleären, auch von spindeliger Form, bestehen,



Fig. 4. Längsschnitt durch den N. trigeminus; un erweiterte Gefäße entwickeltes mykosides Gewebe zwischen Bündeln von Nervenfasern.

Vergr. $1: 70$.

um Gefäße entwickelt, zwischen Nervenbündeln in Längsziugen förmliche Straßen bildend, in das Ganglion sich erstrecken (Textfig. 4). Pyroninfärbung läßt unter den kleineren Uninukleären deutliche Plasmazellen erkennen.

Auf Grund der klinischen Erscheinungen wurde auch der rechte Nervus cruralis untersucht; anch in ihm fanden sich dieselben nur noch weniger intensiv entwickelten Veränderungen, wie in den beschriebenen Nerven. An einem Querschnitt sieht man bei schwacher Vergrößerung (Textfig. 5) an einzelnen Stellen des Perineurilemms und seinen zwischen die Nervenbündel sich verzweigenden fetthaltigen Septa, dann auch im Perineurium, namentlich in seinen Innenschichten, und von da sich auf das Endoneurium fortsetzend, lockere Zellanhäufungen. 
Diese sind namentlich um Blutgefäße entwickelt, bestehen wie andernorts aus den verschiedenen Zellen, wobei das Bindegewebe aufgefasert ist und ein mehr weniger zartes Retikulum bildet. Auch hier treten die großen, protoplasmareichen, bald polygonalen, bald ganz unregelmäßig geformten Zellen besonders hervor; einzelne derselben zeigen bei der Pyronin- oder polychromen Methylenblaufärbung die Reaktionen der Plasmazellen (Textfig. 6). Goldfärbung läßt auch an der veränderten Peripherie der Bündel die Achsenzylinder erḳennen.

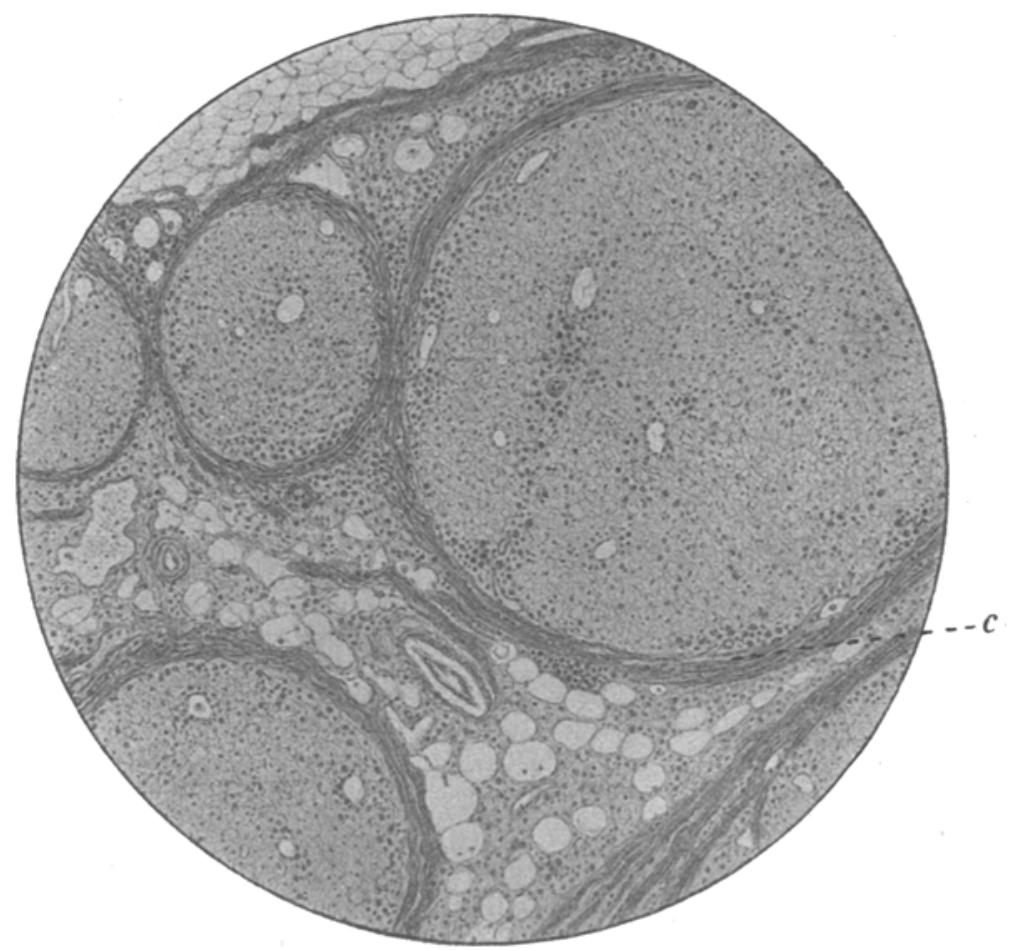

Fig. 5. Aus dem Quersehnitt durch den N. cruralis. Mykosides Gewebe im Perineurilemm, in den Innenschichten des Perineuriums und auch im Endoneurium um Gefäße. Vergr. 1:70.

Die mikroskopische Untersuchung des Knochenmarks vom rechten Oberschenkel erwies dasselbe als Zellmark oder in der Unwandlung zu solchem begriffen; es fanden sich keine mykosiden Veränderungen.

Fassen wir kurz das Ergebnis der histologischen Untersuchung zusammen, so wäre zunächst hervorzuheben, daß außer den Knoten in der Lunge und an der Pleura, den Infiltraten der Magenschleimhaut und dem knotigen Infiltrat im Herzmuskel sich auch die bei der Obduktion vorgefundene Neuritis nervi oculomotorii $d$. sowie des $\mathrm{N}$. trigeminus d. mykosider Natur ist, und daß sich auch im $\mathbb{N}$. cruralis $d$. 
entsprechend den bestandenen Neuralgien in seinem Bereiche beginnende mykoside Veränderungen fanden; daß ferner auch die Schleimhaut des Uterus und das Myometrium, die Sehleimhaut der Harnblase Sitz mykosider Gewebsbildungen sein können, was bisher nicht bekannt war.

Allerorts behält die mykoside Gewebsbildung den Charakter eines Granuloms, dessen Entwicklung mit entzündlichen Erscheinungen, be-



Fig. 6. Die Gegend von $c$ der Fig. 5 bei 320facher Vergrößerung. a Typen der großen, vielgestaltigen, uninukleären Zellen; $b$ Lymphozyten.

trächtlicher Hyperämie, Ödem, auch Ausscheidung von Fibrin bei geringer Zellemigration einsetzt (nicht nur in der Lunge und an der Pleura, sondern. auch im Uterus, Harnblase und Nervengewebe), wobei unter Auffaserung und Auflockerung: des Bindegewebes eigenartige Zellen auftreten, deren Proliferation zum charakteristischen Granulationsgewebe fïhrt.

\section{Tafelerklärung.}

Fig. 1 und 2. Tumoren der Mycosis fungoides an der Pleura diaphragmatica (Fig. 1) und Pleura costalis (Fig. 2). 
Virchorv's Archio Bd. CCXXII.

Iar...

Paltanf nuschertum;

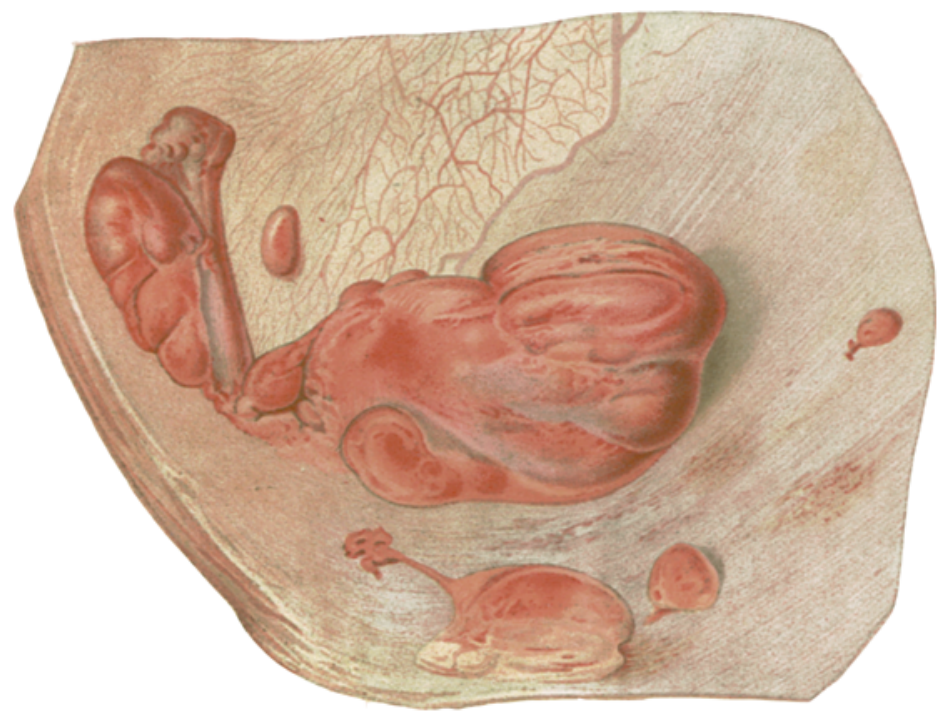

Hig..



Fig. 2. 